\title{
Offline Decoding of Upper Limb Muscle Synergies from EEG Slow Cortical Potentials
}

\author{
Nicolas J. Beuchat, Ricardo Chavarriaga, Sarah Degallier, and José del R. Millán
}

\begin{abstract}
Muscle synergies are thought to be the building blocks used by the central nervous system to control the underdetermined problem of muscles activation. Decoding these synergies from EEG could provide useful tools for BCIcontrolled orthotic devices. In this paper, we assess the possibility of decoding muscle synergies from EEG slow cortical potentials in two healthy subjects and two stroke patients performing a center-out reaching task. We were able to successfully decode the extracted muscle synergies in both healthy subject and one patient.
\end{abstract}

\section{INTRODUCTION}

It has been hypothesized that when moving our limbs, our muscles are controlled as a group , [1]. According to this, a lower dimensional descending neural signal is possibly integrated in the spinal cord before reaching the muscles. This neural signal will thus govern the co-activation of several muscles in what has been termed a muscle synergy. Complex movements are then the result of a weighted combination of a few synergies. Decoding muscle synergies from EEG could be a useful tool to control a robotic prosthesis. Indeed, muscle synergies contain information about the kinematics and dynamics of the arm.

Previous studies have shown that movement parameters, including 3D hand kinematics and onset, can be relatively well decoded from EEG slow cortical potentials (SCPs) [2][3]. We hypothesize that the same signals might contain information about the synergies. In order to test this, we first extract muscle synergies from EMG data of two healthy subjects and two stroke patients during a planar reaching task. We decode the extracted muscle synergies from SCPs using a Linear Decoding Model. We finally compare the decoding performance of muscle synergies and kinematics.

\section{MATERIALS \& METHODS}

\section{A. Experiments}

Two healthy subjects ( 25 and 26 years old) and two stroke patients (Left cerebellar hemorrhagic stroke, left paretic arm, 2 months post-stroke, 50 years old; and left nucleo-capsular stroke, right paretic arm, 2 years post-stroke, 61 years old) were asked to perform center-out planar reaching movements to four targets, $10 \mathrm{~cm}$ away from the center, while holding the

Research supported by the European ICT Programme Project FP7224631, and the SNSF Swiss National Center of Competences in Research (NCCR) Robotics.

N. J. Beuchat, R. Chavarriaga, S. Degallier, J. d. R. Millán are with the Defitech Chair on Non-Invasive Brain-Machine Interface, Center for Neuroprosthetics, Ecole Polytechnique Fédérale de Lausanne, Lausanne, Switzerland. (nicolas.beuchat@gmail.com, jose.millan@epfl.ch).
PHANTOM robotic arm, which recorded the kinematics at $100 \mathrm{~Hz}$. For each arm, subjects performed three runs each containing 80 trials (20 trials per target) (See [3] for details).

Scalp EEG were recorded for 64 electrodes $(10 / 20$ international system) at $2048 \mathrm{~Hz}$. Electrooculograph signals (EOG) were recorded at the same sampling frequency with three electrodes positioned above the nasion, and below the outer canthi of the eyes. Raw EEG and EOG signals were low-pass filtered at $50 \mathrm{~Hz}$ (Butterworth, $4^{\text {th }}$ order, zerophase), resampled to $100 \mathrm{~Hz}$, high-pass filtered at $0.2 \mathrm{~Hz}$ (Butterworth, $4^{\text {th }}$ order, zero-phase), and low-pass filtered at $1 \mathrm{~Hz}$ (Butterworth, $4^{\text {th }}$ order, zero-phase). EEG signals were corrected for EOG activity by removing the best fit of the EOG signals on each EEG channel with multiple linear regression [4]. Each EEG channel was normalized to have zero-mean and unit standard deviation on the whole signal.

EMG signals were recorded at $1 \mathrm{KHz}$ for 16 muscles of the upper arm, forearm, shoulder, upper back and chest. Raw EMGs signals were high-pass filtered at $50 \mathrm{~Hz}\left(50^{\text {th }}\right.$ order FIR, window-based), rectified, low-pass filtered at $20 \mathrm{~Hz}$ ( $50^{\text {th }}$ order FIR, window-based), down-sampled to $100 \mathrm{~Hz}$, detrended and baseline corrected based on resting epochs between trials.

Probably due to muscle fatigue, the EMG baseline level was not constant over the whole experiment in patients. Therefore, we first segmented each run in 3 segments (about 3 min each) and identified rest epochs in each segment using a K-means algorithm $(\mathrm{K}>2)$ [5]. Then, we linearly detrended the signal using a linear regression for each run using only the data points in rest epochs.

After detrending, we removed the baseline muscle activation level for each muscle. We identified the episodes where a muscle was inactive using again a K-means algorithm $(\mathrm{K}>2)$ where each point was clustered by taking a window around it (25 samples). The cluster containing the rest EMG value was identified as the cluster with the smallest centroid norm. Baseline muscle activation was determined by taking the peak of the distribution of the cluster containing rest EMG.

Processed EMGs were further normalized to have unit variance so that synergies extraction would not be biased towards describing only high-amplitude muscles [6],[7].

\section{B. Time-varying synergies}

Muscle activation (EMG) can be represented in a lower dimensional space by the sum of $M$ continuous positive activation coefficients multiplied by their fixed positive weight vector [8]: 


$$
E M G[t]=\sum_{i=1}^{M} c_{i}[t] \vec{w}_{i}+\vec{\varepsilon}
$$

where $M$ is the number of synergies, $c$ the activation coefficient, $w$ the synergies weights, and $\varepsilon$ the residuals.

We estimated the synergies using the Non-Negative Matrix Factorization algorithm using half of the trials as training set [9][10][11]. The number of synergies was chosen so that the reconstruction $\mathrm{R}^{2}$ of the remaining trials exceeded $80 \%$. The procedure was repeated 10 times with different training and testing sets. Once the number of synergies has been identified, the synergies weights and activation coefficients were extracted using all trials [12].

\section{Decoding}

To continuously decode muscle synergies and hand kinematics from EEG signals, we used the following linear decoding model, similar to [2]:

$$
c_{i}[t]=a_{i}+\sum_{n=1}^{N} \sum_{k=0}^{L} b_{n k i} E E G_{n}[t-k]
$$

where $c_{i}$ is the activation coefficient of the $i^{\text {th }}$ synergy, $a$ and $b$ are weights obtained from multiple linear regression, $L$ is the number of lags $(L=10)$, and $N$ is the number of EEG sensors used $(N=16)$. We selected electrodes located bilaterally over the motor cortex (Fz, FC3, FC1, FCz, FC2, $\mathrm{FC} 4, \mathrm{C} 3, \mathrm{C} 1, \mathrm{Cz}, \mathrm{C} 2, \mathrm{C} 4, \mathrm{CP} 3, \mathrm{CP} 1, \mathrm{CPz}, \mathrm{CP} 2$, and CP4).

The same decoding model was used for decoding hand kinematics for each Cartesian coordinate where $c_{i}$ was replaced by the end-effector velocity. In addition, we applied the same technique to decode kinematics from synergies where the EEG signals were replaced by the synergies activation coefficients.

Decoding performance was assessed by an 8 -fold crossvalidation. The performance measure is the Pearson's correlation coefficient between the decoded synergies or kinematics and the actual signal. We computed the chance level by shuffling the input trials for training the decoder. We repeated the procedure 1000 times to get a distribution of decoding performance from random inputs and computed the one-sided $95 \%$ confidence interval as the chance level.

\section{RESULTS}

\section{A. Number of synergies}

The number of synergies required to obtain a reconstruction $\mathrm{R}^{2}$ of $80 \%$ on the test set are given in Table $\mathrm{I}$.

TABLE I. NUMBER OF SYNERGIES

\begin{tabular}{|l|c|c|c|c|}
\hline \multirow{2}{*}{ Arm } & \multicolumn{4}{|c|}{ Number of synergies } \\
\cline { 2 - 5 } & Healthy 1 & Healthy 2 & Patient 1 & Patient 2 \\
\hline Left & 7 & 7 & $10^{\mathrm{a}}$ & 9 \\
\hline Right (dominant) & 7 & 7 & 9 & $9^{\mathrm{a}}$ \\
\hline \multicolumn{4}{|c}{ a. Paretic arm }
\end{tabular}

\section{B. Decoding kinematics from synergies}

We first decoded kinematics from the synergies activation coefficients low-pass filtered at 1, 2, 5, 10, or $20 \mathrm{~Hz}$ (Butterworth, $4^{\text {th }}$ order, zero-phase). This allows us to determine whether high-frequency components of the synergies signals are relevant. The results of this decoding are given in Fig. 1. Filtering at $1 \mathrm{~Hz}$ gave the best decoding accuracy $(\mathrm{p}<0.01$, Paired sample Wilcoxon test, $\mathrm{N}=8)$. Therefore, for the rest of the paper, we use a low-pass filter at $1 \mathrm{~Hz}$ on the synergies signal that we decode from EEG.

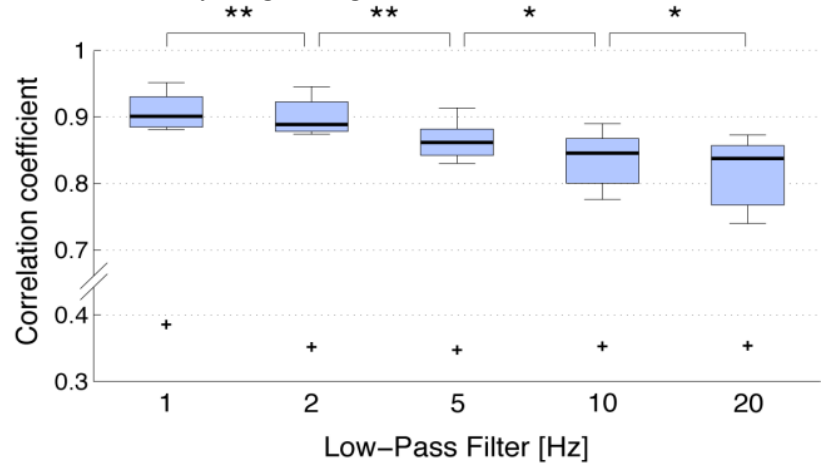

Figure 1. Decoding kinematics from synergies low-pass filtered at different cutoff frequencies. Significant differences were assessed using a Paired sample Wilcoxon test $(\mathrm{N}=8)$. *: $\mathrm{p}<0.05$; ** $\mathrm{p}<0.01$.
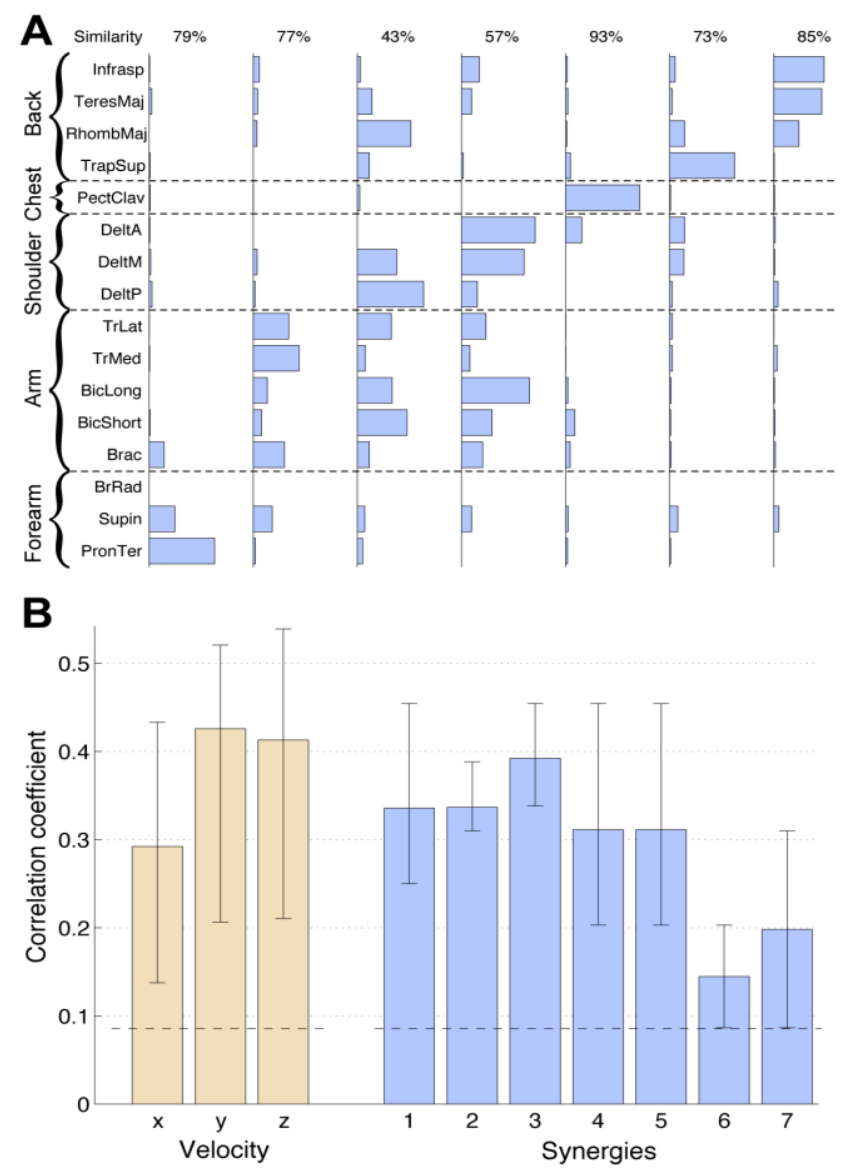

Figure 2. (A) Mean synergies weight coefficients extracted for both arms of the two healthy subjects. Synergies across arms and subjects were matched by normalized dot product similarity and averaged [12]. The similarity for each synergy is indicated above each synergy. Each column corresponds to one synergy and each row to one of the recorded muscles. (B) Mean decoding performance (8-fold CV) of the kinematics and synergies activation coefficients. Error bars indicate the minimum and maximum performance for each decoded signal. 


\section{Decoding synergies and kinematics from EEG slow cortical potentials in healthy subjects}

The mean synergies weights coefficients for the healthy subjects are shown in Fig.2A. We see that synergies weights tend to cluster by anatomical position such as forearm, back, etc. Fig. 2B shows the decoding performance of the kinematics and synergies decoded from EEG slow cortical potentials. In both cases, decoding performances exceeds chance level.

\section{Decoding synergies and kinematics from EEG slow cortical potentials in stroke patients}

The extracted synergies weight coefficient for the paretic and non-paretic arms as well as the decoding performance of the kinematics and synergies activation coefficients of two stroke patients are presented in Fig. 3.

\section{DISCUSSION \& CONCLUSION}

This pilot study provides evidence on the possibility of decoding muscle synergies from EEG SCPs. We report results with two healthy subjects and two stroke patients performing a planar center-out reaching task. This allows us to analyze this task at different levels: cortical, muscular, and kinematic. It also allows us to understand what information is conveyed from one level to the next.

Regarding kinematics decoding performances, our results were on average slightly higher $\left(r_{x}=0.29, r_{y}=0.43, r_{z}=0.41\right)$ than previous studies [2] $\left(r_{x}=0.19, r_{y}=0.32, r_{z}=0.38\right)$ and [13] $\left(r_{x}=0.37, r_{y}=0.24\right)$.

Notably, we showed that in healthy subjects, the decoding of most muscle synergies exceeds chance level and yields performances close to that of the kinematics. The lowest classification (slightly higher than chance level), was obtained for the synergies controlling the back muscles.

The extracted synergies in healthy subjects were stable across subjects (normalized dot product similarity of $73 \%$ or higher for 5 synergies) except for the synergies controlling the shoulder, flexor, and extensor muscles where the similarity was less than $60 \%$.

In the case of stroke patients, the decoding of the kinematics and synergies in the paretic arm of the patient with a left cerebellar hemorrhagic stroke was below chance level. Surprisingly, the decoding performance of the synergies in the non-paretic arm was higher than chance whereas the decoding of the kinematics was not. The very low decoding performance might be explained by the short time between the lesion and the experiment. For one patient with a left nucleo-capsular stroke, we have shown that the decoding of the kinematics is in the same range as for healthy subjects for both the paretic and non-paretic arm. For the synergies decoding, the performance were slightly lower than for healthy subject (paretic arm: $r=0.09-0.29$, nonparetic arm: $\left.r_{\min }=0.01-0.21\right)$. However, the number of synergies for patients was higher than for healthy subjects even for the non-paretic arm (3-4 additional synergies). This makes the comparison of synergies decoding difficult between patients and healthy subjects. In addition, the oneto-one comparison of synergies decoding in patients might not be extremely relevant due to possible splitting and merging of synergies in the paretic arm [12].

We note that we only used 16 EEG channels on the motor cortex to decode both kinematics and synergies. In addition, we only used slow cortical potentials as a first attempt to decode synergies. Different frequency bands might be involved in the encoding of muscle synergies.

In the future, we will assess kinematics decoding from the subset of the best decoded synergies. This would indicate which synergies are actually important to be decoded from EEG. In addition, we will test if similar subsets of synergies across subjects and limbs systematically yield comparable decoding accuracy. For example, we see from this two subjects analysis that the forearm, extensor, shoulders and flexors, and chest synergies are relatively well decoded $(r=0.34,0.34,0.39,0.31$, and 0.31 respectively) while the synergies controlling the back muscles are not $(r=0.14$ and 0.2 ). If further studies confirm this trend, this subset of synergies can be used as control input to to an exoskeleton or FES orthosis [14].

\section{ACKNOWLEDGMENT}

The authors thank Dr. S. Silvoni (Ospedale San Camillo, Venezia, Italy) and Luca Tonin (EPFL) for initial work and data collection.

\section{REFERENCES}

[1] N. A. Bernstein, The Co-ordination and regulation of movements. New York: Pergamon, 1967.

[2] T. J. Bradberry, R. J. Gentili, and J. L. Contreras-Vidal, "Reconstructing three-dimensional hand movements from noninvasive electroencephalographic signals," J. Neurosci., vol. 30, no. 9, pp. 3432-3437, 2010.

[3] E. Lew, R. Chavarriaga, S. Silvoni, and J. del R. Millán, "Detection of self-paced reaching movement intention from EEG signals," Front. Neuroeng., vol. 5, 2012.

[4] A. Schlögl, C. Keinrath, D. Zimmermann, R. Scherer, R. Leeb, and G. Pfurtscheller, "A fully automated correction method of EOG artifacts in EEG recordings," Clinical Neurophysiology, vol. 118, no. 1, pp. 98-104, Jan. 2007.

[5] G. A. F. Seber, Multivariate observations. Hoboken, NJ: John Wiley \& Sons, Inc., 1984.

[6] G. Torres-Oviedo, J. M. Macpherson, and L. H. Ting, "Muscle synergy organization is robust across a variety of postural perturbations," J. Neurophysiol., vol. 96, no. 3, pp. 1530-1546, 2006.

[7] V. C. K. Cheung, A. d'Avella, and E. Bizzi, "Adjustments of motor pattern for load compensation via modulated activations of muscle synergies during natural behaviors," J. Neurophysiol., vol. 101, no. 3, pp. 1235-1257, 2009.

[8] V. C. K. Cheung, A. d'Avella, M. C. Tresch, and E. Bizzi, "Central and sensory contributions to the activation and organization of muscle synergies during natural motor behaviors," J. Neurosci., vol. 25, no. 27, pp. 6419-6434, 2005.

[9] P. Paatero and U. Tapper, "Positive matrix factorization: A nonnegative factor model with optimal utilization of error estimates of data values," Environmetrics, vol. 5, no. 2, pp. 111-126, Jun. 1994.

[10] D. D. Lee and H. S. Seung, "Learning the parts of objects by nonnegative matrix factorization," Nature, vol. 401, no. 6755, pp. 788791, 1999.

[11] M. W. Berry, M. Browne, A. N. Langville, V. P. Pauca, and R. J. Plemmons, "Algorithms and applications for approximate nonnegative matrix factorization," Computational Statistics \& Data Analysis, vol. 52, no. 1, pp. 155-173, 2007. 

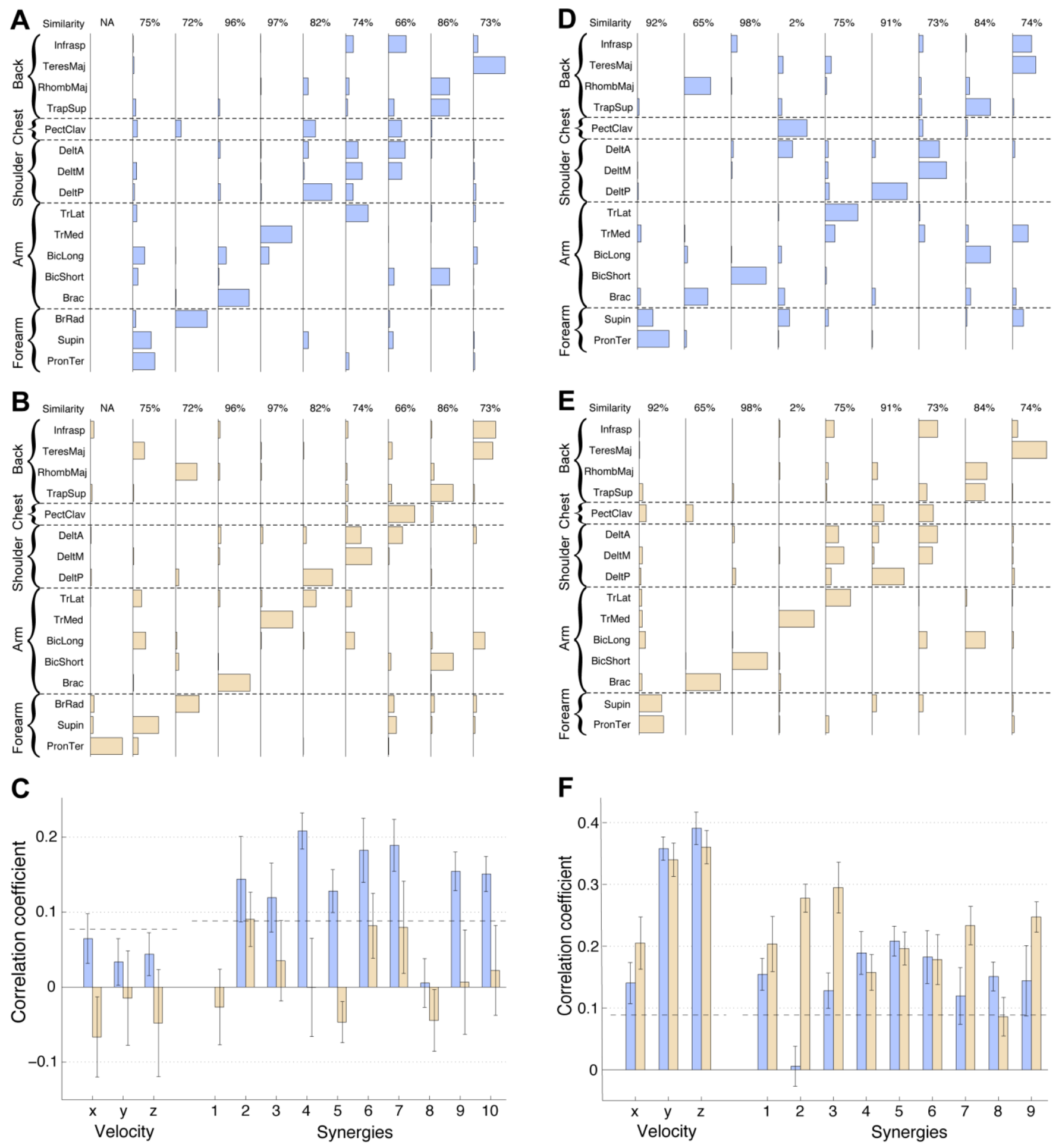

Figure 3. Synergies extracted for the non-paretic arm (Top) and paretic arm (Middle) of two stroke patients. Synergies of the paretic and non-paretic arms were matched givng the highest dot product (indicated above each synergies). (Bottom) Decoding performance of the kinematics and synergies decoding from EEG for the non-paretic (blue) and paretic arm (beige). (Left column) Patient with a left cerebellar hemorragic stroke (left paretic arm, non-dominant, 2 months post-stroke). (Right column) Patient with a left nucleo-capsular stroke (right paretic arm, dominant, 2 years post-stroke).

[12] V. C. K. Cheung, A. Turolla, M. Agostini, S. Silvoni, C. Bennis, P. Kasi, S. Paganoni, P. Bonato, and E. Bizzi, "Muscle synergy patterns as physiological markers of motor cortical damage," Proc Nat Academy of Sciences, vol. 109, no. 36, pp. 14652-14656, 2012.

[13] J. Lv, Y. Li, and Z. Gu, "Decoding hand movement velocity from electroencephalogram signals during a drawing task," BioMed Eng OnLine, vol. 9, no. 1, p. 64, 2010.
[14] J. del R. Millán, R. Rupp, G. R. Müller-Putz, R. Murray-Smith, C. Giugliemma, M. Tangermann, C. Vidaurre, F. Cincotti, A. Kübler, and R. Leeb, "Combining brain-computer interfaces and assistive technologies: state-of-the-art and challenges," Front neurosci, vol. 4, 2010. 